\title{
Immunodetection of the MCHR1 antibody in vitiligo patient sera
}

\author{
MIAONI ZHOU ${ }^{1}$, CUIPING GUAN ${ }^{1}$, FUQUAN LIN $^{1}$, WEN XU ${ }^{1}$, LIFANG FU ${ }^{1}$, \\ WEISONG HONG ${ }^{1}$, YINSHENG WAN ${ }^{2}$ and $\mathrm{AIE} \mathrm{XU}^{1}$ \\ ${ }^{1}$ Department of Dermatology, The Third People's Hospital of Hangzhou, Hangzhou 310009, P.R. China; \\ ${ }^{2}$ Department of Biology, Providence College, Providence, RI 02918, USA
}

Received October 20, 2010; Accepted December 29, 2010

DOI: $10.3892 / \mathrm{ijmm} .2011 .629$

\begin{abstract}
Human vitiligo is an acquired depigmenting skin disorder characterized by milk-white skin macules resulting from a chronic and progressive loss of melanocytes. It has been suggested that autoimmune mechanisms are involved in this disorder. We undertook this project to obtain an MCHR1-C linear peptide containing four main MCHR1 B-cell epitopes in an attempt to detect the IgG antibody against MCHR1 in vitiligo. The target gene encoding the MCHR1-C peptide was cloned into a pGEX-4T-2 expression vector, expressed in Escherichia coli BL21, and purified using a GST column. The molecular weight was analyzed by SDS-PAGE and Western blotting. The IgG antibody response to MCHR1 was detected by ELISA with MCHR1-C as a coated antigen, and the absorption experiment was used to assess the binding ability of the Ab detected by MCHR1-C with a membrane protein in human epidermal melanocytes. The purified peptide MCHR1-C with a molecular weight of $33 \mathrm{kDa}$ was obtained, and could bind to the MCHR1 antibody in human sera. Of the vitiligo patient sera examined, 24 of 145 (16.55\%) tested positive for the MCHR1 antibody, and the frequency in the vitiligo patient group was significantly greater compared to the normal control. However, no significant difference between gender, disease duration, clinical subtype or family history between the two groups was observed. In addition, the $\mathrm{Ab}$ detected by MCHR1-C in sera was specifically absorbed by the membrane protein obtained from human epidermal melanocytes. Collectively, our data suggest that the MCHR1-C peptide can be used to detect the MCHR1 antibody in vitiligo patients as a coated antigen instead of MCHR1 protein. We conclude that the level of MCHR1 antibody in active vitiligo patients is significantly higher than that in healthy individuals, while gender, disease duration, clinical subtype and family
\end{abstract}

Correspondence to: Dr Aie Xu, Department of Dermatology, The Third People's Hospital of Hangzhou, Hangzhou 310009, P.R. China E-mail: xuaiehz@msn.com

Dr Yinsheng Wan, Department of Biology, Providence College, RI 02918, USA

E-mail: yswan@providence.edu

Key words: melanin-concentrating hormone receptor 1, vitiligo, autoantibody, expression peptide history have no association with the level of MCHR1 antibody in vitiligo patients.

\section{Introduction}

Vitiligo is a disorder of pigmentation with a prevalence of $1-4 \%$. It is an acquired idiopathic hypomelanotic disorder characterized by circumscribed white macules resulting from a chronic and progressive loss of cutaneous melanocytes. Although autoimmunity has been suggested to play a role in the development of the disease (1-3), the pathogenesis of vitiligo remains obscure. The characterization of melanocyte autoantigens in vitiligo is essential for understanding the immunopathological mechanisms. Previous studies have revealed that autoantibodies in vitiligo patients are commonly directed against melanocyte antigens that are located on the cell surface (4) and some specific vitiligo-associated autoantigens (5-8) have been successfully identified.

Melanin-concentrating hormone receptor 1 (MCHR1) has been characterized as an important B-cell autoantigen located on the plasma membrane of melanocytes. The MCHR1 autoantibody can block the response of the receptor to the melanin-concentrating hormone (MCH) (9). Recently, vitiligo has been reported to react against multiple epitopes on MCHR1, and major autoantibody epitopes are localized in the 51-80, 85-98, 154-158 and 254-260 peptides (10).

In this study, an MCHR1-C linear peptide containing the MCHR1 epitopes 51-80, 85-98, 154-158 and 254-260 was expressed in vitro. The autoantibody response to MCHR1 was detected with ELISA using the peptide MCHR1-C in vitiligo patients. The specificity of the antibody binding to MCHR1 in positive vitiligo sera were assessed by absorption experiment, and the variables including gender, duration, clinical subtypes, and family history were also compared between antibody-positive and antibody-negative vitiligo patients.

\section{Materials and methods}

Subjects. Sera from 145 active vitiligo patients without other autoimmune disorders (67 male, 78 female; mean age, 28.7 years; age range, 6-70 years) and 72 healthy individuals (32 male, 40 female; mean age, 31.3 years; age range, 18-72 years) were collected in this study. Among these patients, there were 22 localized, 46 sporadic, 7 generalized, 30 acromelic and sporadic, 13 acromelic and 27 segmental cases, and 15 patients 
had a family history of vitiligo. In addition, sera from 20 atopic dermatitis (AD) patients and 17 Graves' disease (GD) patients were collected. All sera were centrifuged and kept frozen at $-20^{\circ} \mathrm{C}$. The approval of the local ethics committee was obtained for the study and all subjects gave informed consent.

Construction of the expression vector. The nucleotide sequence encoding the main B-cell epitopes (amino acids 51-80, 85-98, 154-158, 254-260) of MCHR1 was chemically synthesized according to the cDNA of MCHR1 (NM005297.3), and the restriction sites for $\mathrm{BamHI}$ and $\mathrm{XhoI}$ were incorporated to the 5'- and 3'-ends, respectively (Jinsite Biotechnology, China). The synthesized gene was cloned into the BamHI and XhoI sites of the prokaryotic expression vector pGEX-4T-2 (a gift from Professor Chuanxi Zhang, Institute of Insect Sciences, Zhejiang University, China). The recombinant plasmids named pGEX-MCHR1-C were confirmed by restriction analysis and sequencing.

Prokaryotic expression. The correct recombinant expression vectors were transformed into Escherichia coli BL21 for expression. A single colony of $E$. coli BL21 was inoculated into LB medium supplemented with ampicillin $(100 \mu \mathrm{g} / \mathrm{ml})$, and cultured at $37^{\circ} \mathrm{C}$ with shaking $(250 \mathrm{rpm})$ until optical density $(\mathrm{OD})_{600} \sim 0.4$. Then the expression was induced with isopropy- $\beta$-D-thiogalactoside (IPTG) (Sangon, Shanghai, China) to a final concentration of $1.0 \mathrm{mmol} / 1$ at $37^{\circ} \mathrm{C}$ in an incubator shaker. The bacteria were harvested after $8 \mathrm{~h}$ and lysed by ultrasonic disruption for three times with pulse and interval times of $5 \mathrm{sec}$ each. The whole process was operated in an ice bath. Disrupted cells were centrifuged at 12,000 rpm for $10 \mathrm{~min}$ at $4^{\circ} \mathrm{C}$ to separate soluble proteins and insoluble pellets. After centrifugation, the clear lysates were obtained and stored at $-20^{\circ} \mathrm{C}$. The recombinant proteins were purified using GST resin (Pierce, USA) according to the manufacturer's protocol. The size and quantity of the expressed target protein was determined by $12 \%$ SDS-PAGE.

Sodium dodecyl sulfate polyacrylamide gel electrophoresis (SDS-PAGE) and protein analysis. Protein analysis was performed on $12 \%$ SDS-PAGE gels. Protein samples were boiled for $5 \mathrm{~min}$ under denaturing conditions and then centrifuged at $10,000 \mathrm{x}$ g for $5 \mathrm{~min}$ prior to loading on the gel. Protein concentrations in the samples were determined by the Bradford method with bovine serum albumin as a standard. Protein purity was estimated by densitometric analysis of protein bands separated by $12 \%$ SDS-PAGE after staining with Coomassie brilliant blue R-250 (Beyotime, China).

ELISA detection. The ELISA plates were coated overnight at $4^{\circ} \mathrm{C}$ with $100 \mu 1$ of MHCR1-C with the optimized concentrations at $0.125 \mu \mathrm{g} / \mathrm{ml}$. Plates were washed in phosphate-buffered saline, pH 7.2, containing 0.5\% (vol/vol) Tween-20 (PBST). After five washes with PBST, each well was blocked with $5 \%$ skim milk for $1 \mathrm{~h}$ at $37^{\circ} \mathrm{C}$. The plate was washed again, followed by the addition of serum samples (diluted 1:10), and incubated at $37^{\circ} \mathrm{C}$ for $1 \mathrm{~h}$. After the excess serum samples were washed off, mouse anti-human IgG-HRP (Promega, USA) (diluted in PBST, 1:5000) was added and incubated at $37^{\circ} \mathrm{C}$ for $1 \mathrm{~h}$. Following a final washing step, $50 \mu \mathrm{l}$ of tetramethyl benzidine (TMB) substrate solution (Promega, USA) was added. OD was measured at $450 \mathrm{~nm}$ wavelength after stopping the reaction with $50 \mu \mathrm{l}$ of $0.5 \mathrm{M} \mathrm{H}_{2} \mathrm{SO}_{4}$. The ODs were measured using an ELISA spectrophotometer. Each serum was tested in three independent experiments. The upper level of normal for the assay was the mean plus three standard deviations (SD) of the mean OD of 30 serum samples from healthy individuals. Any serum sample with OD above the upper level was designated as positive. The frequency of Ab's was compared between vitiligo patients and healthy individuals using a $\chi^{2}$ test, with $\mathrm{P}<0.05$ regarded as significant. The characteristics of the Ab-positive and Ab-negative vitiligo patients were also compared using the $\chi^{2}$ test, with $\mathrm{P}<0.05$ regarded as significant. For dilution experiments, each positive vitiligo serum was used in ELISA at final dilutions of 1:10, 1:20, 1:40, 1:80, 1:160 and 1:320.

Absorption experiment. Normal human epidermal melanocytes were obtained from full-thickness foreskin and cultured in HU-16 medium. Cell membrane proteins were prepared using a membrane protein extraction kit (Biovision, USA). Sera positive for MCHR1 IgG antibody and healthy controls (diluted 1:20) were incubated with membrane proteins of melanocytes $(30 \mu \mathrm{g})$ at $4^{\circ} \mathrm{C}$ overnight. After pre-incubation, the sera were used in ELISA. Sera without prior absorption were assayed concurrently. The difference of OD-values between the two groups was analyzed using the Student's t-test, with $\mathrm{P}<0.05$ regarded as significant.

\section{Results}

Construction of the recombinant plasmid. The nucleotide sequence encoding MCHR1-C was inserted downstream of the GST-encoding DNA in the same open reading frame at the $B a m \mathrm{HI}$ and EcoRI restriction sites of the multiple cloning sites of pGEX-4T-2. The correct expression vector pGEX-MCHR1-C was confirmed by restriction enzyme analysis and PCR-sequencing. After the expression vector was digested by BamHI and EcoRI, a fragment with a size of $174 \mathrm{bp}$ (Fig. 1), which was the same as the predicted size of the target gene, was detected. The results indicate that the nucleotide sequence encoding MCHR1-C was inserted into multiple cloning sites of pGEX-4T-2.

Expression and purification of the linear peptide MCHR1-C. After IPTG induction, E. coli BL21 transformed with the pGEX-4T-2 vector produced a protein of $26 \mathrm{kDa}$ (GST protein) (Fig. 2, lane 3). Similarly, E. coli BL21 transformed with the pGEX-MCHR1-C produced a MCHR1-C (GST fusion protein) protein of around $33 \mathrm{kDa}$ (Fig. 2, lane 5), and the size of the expressed protein matched well with its theoretical molecular mass. In contrast, non-induced E. coli BL21 cells did not express this protein. According to the densitometric scanning of the stained gel, soluble GST-MCHR1-C fusion protein accounted for approximately $60 \%$ of total expressed target proteins (Fig. 2, lanes 5 and 6). The soluble fusion protein was purified with a GST column purification kit, with $90 \%$ purity. These results indicate that the recombinant MCHR1-C was successfully expressed in the constructed E. coli BL21. 


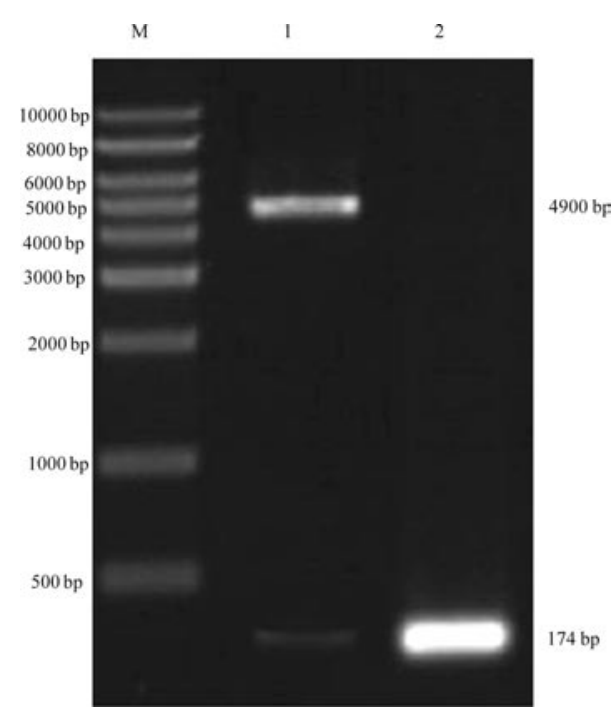

Figure 1. Restrictive digestion mapping of the recombinant expression vector. Lane M, KB ladder marker; lane 1, Bam HI/XhoI restricted plasmid pGEX-MCHR1-C; lane 2, amplified PCR product of the target gene.

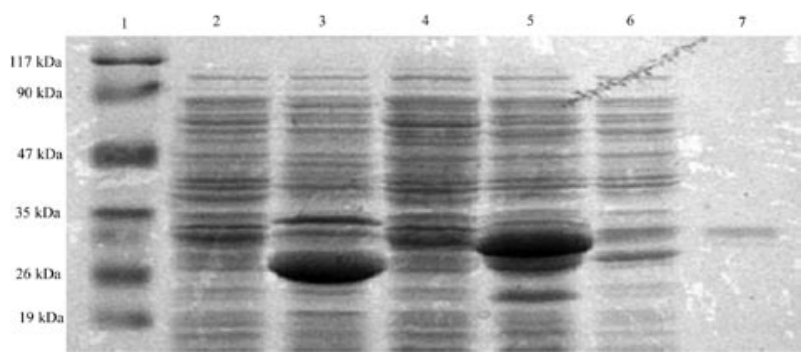

Figure 2. SDS-PAGE of the expressed MCHR-C protein. Lane 1, protein marker; lane 2, expressed protein of Escherichia coli BL21 (negative control); lane 3, expressed protein of pGEX-4T-2 (positive control); lane 4, expressed protein of pGEX-MCHR1-C without IPTG induction; lane 5, the total expressed protein of pGEX-MCHR1-C with IPTG induction; lane 6, expressed hydrophilic protein of pGEX-MCHR1-C with IPTG induction; lane 7 , target protein purified with GST.

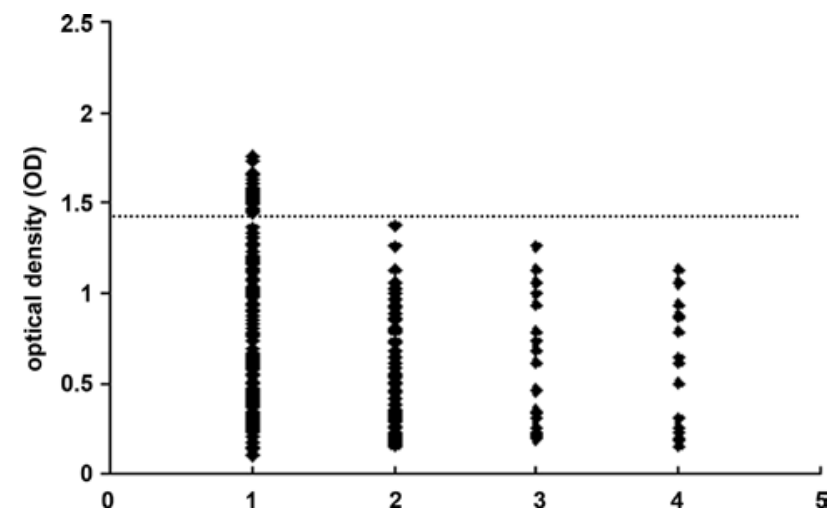

Figure 3. ELISA assays. Lane 1, vitiligo patients; lane 2, healthy controls lane 3, AD patients; lane 4, GD patients. Sera from 145 active vitiligo patients, 72 healthy controls, $20 \mathrm{AD}$ patients and 17 GD patients were examined. The OD-value shown is the mean of two experiments. The broken line shows the upper level of normal at an OD-value of 1.427.

Detection of autoantibody to MCHRl in human sera. Sera from 145 vitiligo patients, 72 healthy controls, $20 \mathrm{AD}$ and

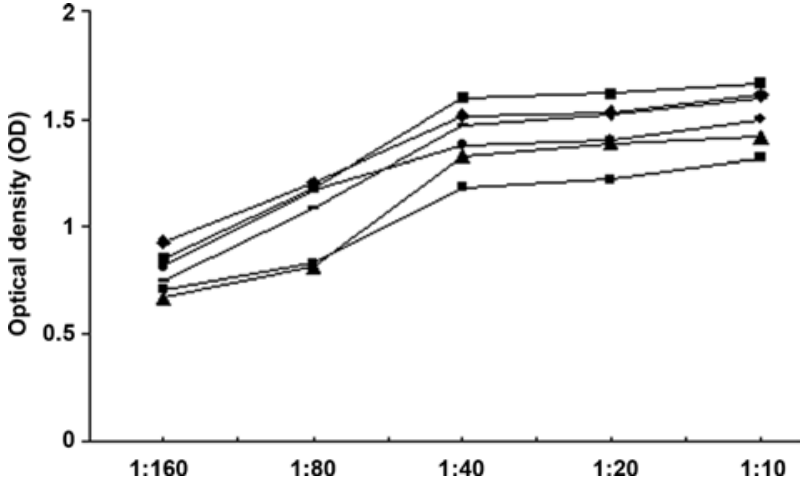

Figure 4. Dilution curves of six antibody positive sera. The OD-value of each positive serum at each dilution is plotted as a function of serum dilution. Each serum was analyzed in three experiments and the mean OD-value was calculated.

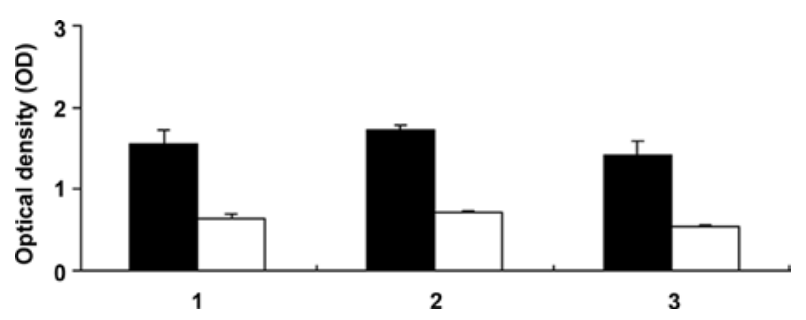

Figure 5. Absorption experiment. MCHR1 Ab-positive sera $(n=3)$ were preabsorbed with the membrane protein from human epidermal melanocytes before analysis with ELISA. Samples without pre-absorption shown with black bars; samples with pre-absorption shown with white bars.

17 GD patients were analyzed. Each serum sample was tested in three experiments. The upper level of normal (mean OD-value plus 3SD of 72 healthy controls) was established as OD-value of 1.427. Any serum with an OD-value $>1.427$ was considered positive for MCHR1 Ab's. According to this standard, sera from 72 healthy controls, $20 \mathrm{AD}$ patients and 17 GD patients were all negative for Ab's to MCHR1, and 24 of 145 patients (16.55\%) were considered positive (Fig. 3). The difference between the vitiligo patient group and the normal control was significant $\left(\chi^{2}=13.399, \mathrm{P}<0.001\right)$. In dilution experiments of all positive sera, three were positive at a maximal dilution of 1:40, three were positive at a maximal dilution of 1:20, and the other sera were positive at a final dilution of 1:10. According to the OD-values of six positive sera at every dilution (Fig. 4), saturated binding was observed at dilutions up to 1:40.

Absorption experiment. Antibody-positive vitiligo sera $(\mathrm{n}=3)$ were pre-incubated with the membrane protein isolated from human epidermal melanocytes, followed by ELISA analysis as described above. Each serum was tested three times. The mean OD-value for each positive serum without pre-incubation with the membrane protein of human epidermal melanocytes was $1.546 \pm 0.177,1.729 \pm 0.059$ and $1.432 \pm 0.156$, respectively (Fig. 5, black bars). After pre-incubation with the membrane protein, the mean OD-value of each positive serum was $0.651 \pm 0.006,0.715 \pm 0.037,0.538 \pm 0.023$, respectively (Fig. 5, white bars). The OD-values were significantly decreased in the serum samples pre-absorbed with the membrane protein 
Table I. Characteristics of the MCHR1 Ab-positive and Ab-negative patients.

Vitiligo patients $(n=145)$

Ab-positive $(n=24)$

Ab-negative $(n=121)$

\begin{tabular}{lcc}
\hline Gender & & \\
Male & $11(45.8 \%)$ & $56(46.3 \%)$ \\
Female & $13(54.2 \%)$ & $65(53.7 \%)$ \\
Disease duration & & \\
$<5$ years & $17(70.8 \%)$ & $81(66.9 \%)$ \\
$\geq 5$ years & $7(29.2 \%)$ & $40(33.1 \%)$ \\
Mean age (range) & $36.4(6-70)$ years & $21.6(6-63)$ years \\
Vitiligo subtypes & & \\
Localized & $4(16.6 \%)$ & $18(14.9 \%)$ \\
Sporadic & $5(20.8 \%)$ & $41(33.9 \%)$ \\
Generalized & $1(4.2 \%)$ & $6(4.9 \%)$ \\
Acromelic and sporadic & $3(12.55 \%)$ & $27(22.3 \%)$ \\
Acromelic & $2(8.3 \%)$ & $11(9.1 \%)$ \\
Segmental & $9(37.5 \%)$ & $18(14.9 \%)$ \\
Family history & & $10(8.3 \%)$ \\
Yes & $5(20.8 \%)$ & $111(91.7 \%)$ \\
No & $19(79.2 \%)$ &
\end{tabular}

of human epidermal melanocytes $(\mathrm{P}<0.001)$. The results suggest that the IgG antibody, which was detected by the linear recombinant antigen MCHR1-C in the vitiligo patients, could be specifically absorbed by MCHR1 protein in human epidermal melanocytes.

Comparison of MCHRl Ab-positive and negative vitiligo patients. The characteristics of MCHR1 Ab-positive and negative vitiligo patients, including gender, disease duration, mean age, vitiligo subtypes and family history, are summarized in Table I. The differences in gender, disease duration, vitiligo subtype and family history between the antibodypositive and antibody-negative groups were analyzed using the Fisher's exact test, and P-values were 0.968, 0.710, 0.065 and 0.180 , respectively. The results indicate that there is no association between the presence of MCHR1 antibody and gender, disease duration, clinical subtype, or family history.

\section{Discussion}

Vitiligo is a disorder of pigmentation and little is known about its aetiology. One of the most popular hypotheses considers vitiligo as an autoimmune disease. Previous investigations have proven that the anti-melanocyte antibody, which destroys the skin melanocyte, is one of the important autoantibodies causing vitiligo (11-13). The important antigens detected in vitiligo patients include, but may not be limited to, tyrosinase (5), tyrosinase-related protein-1 (TRP-1) (7), tyrosinaserelated protein-2 (TRP-2) (6), Pmel17 (14), the transcription factor SOX10 (8) and MCHR1 (9). Of these autoantigens, MCHR1 is the sole antigen on the cell surface, although antibodies in vitiligo patients are most commonly directed against melanocyte antigens on the cellular membrane (4).

MCHR1 is a G protein-coupled receptor for the neuropeptide $\mathrm{MCH}$. The function of the $\mathrm{MCH} / \mathrm{MCHR} 1$ signaling pathway is to mobilize intracellular $\mathrm{Ca}^{2+}$ and reduce forskolin-elevated cAMP levels $(15,16)$. Previous studies revealed that $\mathrm{MCH}$ is a functional antagonist of $\alpha$-melanocyte-stimulating hormone $(\alpha-\mathrm{MSH})$ and could partially inhibit the induction of melanogenesis by $\alpha-\mathrm{MSH}$ in human melanocytes $(15,16)$. MCHR1 expression has been reported in human melanocytes $(17,18)$. Recently, MCHR1 has been suggested as a novel antigen with high specificity in vitiligo (9). In addition, four epitope domains (amino acids 51-80, 85-98, 154-158, 254-260) have been identified as the antibody binding sites (10). Also, the equivalent peptide sequence to epitopes 51-80 and 85-98, 154-158 and 254-260 in rat MCHR1 are predicted to lie in the extracellular $\mathrm{N}$-terminal domain, the first extracellular loop, and the second extracellular loop of the receptor, respectively (19).

In this study, we adopted E. coli BL21 as host bacteria, a protease-deficient strain, and chose the pGEX-4T-2 expression vector containing the GST expression tag to express a novel linear peptide MCHR1-C. In this expression system, soluble GST fusion protein MCHR1-C was obtained with $90 \%$ purity and the molecular weight of MCHR1-C was about $33 \mathrm{kDa}$, while the full-length MCHR1 protein failed to be expressed in this system. MCHR1-C is composed of four main epitopes, 51-80, 85-98, 154-158 and 254-260 of MCHR1.

It has been previously reported that the antibody to MCHR1 was detected in $16.4 \%$ of vitiligo patient sera using the MCHR1 protein expressed in vitro, whereas AD, GD, sytem lupus erythematosus patient and control sera showed 
no reactivity (9). The antibody reactivity in the vitiligo patient sera was specifically absorbed by expressed MCHR1 in the CHO cell membranes (9). In this study, a novel expression peptide MCHR1-C was used in serological ELISA assay as a coated antigen to examine sera from 145 active vitiligo patients, including 72 healthy individuals, $20 \mathrm{AD}$ patients and 17 GD patients. Twenty-four of 145 (16.55\%) patients tested positive for the MCHR1 antibody, while none of the healthy individuals and the other patients with autoimmune diseases (AD and GD) were considered positive. In the dilution experiment, the serum was considered positive at the highest dilution of 1:40, and positive sera showed saturated binding up to a dilution of 1:40. In absorption experiments, the IgG antibody, detected by the linear recombinant antigen MCHR1-C in the vitiligo patients, could be specifically absorbed by MCHR1 protein in the human epidermal melanocytes.

In summary, the expression peptide MCHR1-C can be used to detect the MCHR1 antibody as a coated antigen in ELISA. The incidence of MCHR1 antibody is detectable by MCHR1-C in the same way as full-length MCHR1. Our results indicate that the ELISA assay using MCHR1-C as antigen has a high disease-associated specificity and sensitivity. In addition, the expression of MCHR1-C is more convenient than full-length MCHR1. Hence, as a novel candidate of MCHR1, the expression peptide MCHR1-C has the potential to estimate the level of MCHR1 antibody in vitiligo patients.

\section{Acknowledgements}

This research was supported by grants from Research Funds of the Health Department (200820274) and a grant from NIH (P20 RR016457 from INBRE Program of the National Center for Research Resources). We thank Professor Chuanxi Zhang (Institute of Insect Sciences, Zhejiang University, China) for supplying pGEX-4T-2 and E. coli BL21.

\section{References}

1. Kemp EH, Waterman EA and Weetman AP: Autoimmune aspects of vitiligo. Autoimmunity 34: 65-77, 2001.

2. Le Poole IC and Luiten RM: Autoimmune etiology of generalized vitiligo. Curr Dir Autoimmun 10: 227-243, 2008.

3. Rezaei N, Gavalas NG, Weetman AP and Kemp EH: Autoimmunity as an aetiological factor in vitiligo. J Eur Acad Dermatol Venereol 21: 865-876, 2007.
4. Park YK, Kim NS, Hann SK and Im S: Identification of autoantibody to melanocytes and characterization of vitiligo antigen in vitiligo patients. J Dermatol Sci 11: 111-120, 1996.

5. Baharav E, Merimski O, Shoenfeld Y, et al: Tyrosinase as an autoantigen in patients with vitiligo. Clin Exp Immunol 105: 84-88, 1996.

6. Okamoto T, Irie RF, Fujii S, et al: Anti-tyrosinase-related protein-2 immune response in vitiligo patients and melanoma patients receiving active-specific immunotherapy. J Invest Dermatol 111: 1034-1039, 1998.

7. Kemp EH, Waterman EA, Gawkrodger DJ, et al: Autoantibodies to tyrosinase-related protein-1 detected in the sera of vitiligo patients using a quantitative radiobinding assay. Br J Dermatol 139: 798-805, 1998.

8. Hedstrand H, Ekwall O, Olsson MJ, et al: The transcription factors SOX9 and SOX10 are vitiligo autoantigens in autoimmune polyendocrine syndrome type I. J Biol Chem 276: 35390-35395, 2001.

9. Kemp EH, Waterman EA, Hawes BE, et al: The melaninconcentrating hormone receptor 1 , a novel target of autoantibody responses in vitiligo. J Clin Invest 109: 923-930, 2002.

10. Gavalas NG, Gottumukkala RV, Gawkrodger DJ, et al: Mapping of melanin-concentrating hormone receptor $1 \mathrm{~B}$ cell epitopes predicts two major binding sites for vitiligo patient autoantibodies. Exp Dermatol 18: 454-463, 2009.

11. van den Wijngaard R, Wankowicz-Kalinska A, Pals S, et al: Autoimmune melanocyte destruction in vitiligo. Lab Invest 81: 1061-1067, 2001.

12. Kemp EH, Gavalas NG, Gawkrodger DJ and Weetman AP: Autoantibody responses to melanocytes in the depigmenting skin disease vitiligo. Autoimmun Rev 6: 138-142, 2007.

13. Wang X and Erf GF: Melanocyte-specific cell mediated immune response in vitiliginous Smyth line chickens. J Autoimmun 21: 149-160, 2003.

14. Kemp EH, Gawkrodger DJ, Watson PF and Weetman AP: Autoantibodies to human melanocyte-specific protein pmell7 in the sera of vitiligo patients: a sensitive and quantitative radioimmunoassay (RIA). Clin Exp Immunol 114: 333-338, 1998.

15. Chambers J, Ames RS, Bergsma D, et al: Melanin-concentrating hormone is the cognate ligand for the orphan G-protein-coupled receptor SLC-1. Nature 400: 261-265, 1999.

16. Eberle AN, Mild G, Schlumberger S, et al: Expression and characterization of melanin-concentrating hormone receptors on mammalian cell lines. Peptides 25: 1585-1595, 2004.

17. Schlumberger SE, Talke-Messerer C, Zumsteg U and Eberle AN: Expression of receptors for melanin-concentrating hormone $(\mathrm{MCH})$ in different tissues and cell lines. J Recept Signal Transduct Res 22: 509-531, 2002.

18. Hoogduijn MJ, Ancans J, Suzuki I, et al: Melanin-concentrating hormone and its receptor are expressed and functional in human skin. Biochem Biophys Res Commun 296: 698-701, 2002.

19. Saito Y, Tetsuka M, Saito S, et al: Arginine residue 155 in the second intracellular loop plays a critical role in rat melaninconcentrating hormone receptor 1 activation. Endocrinology 146: 3452-3462, 2005 